\title{
A COMPARISON OF ALTERNATIVE ORDERED RESPONSE MODELS FOR ANALYSING USER SATISFACTION WITH TRANSPORT SERVICES IN TURKEY
}

\author{
Ali Kemal Çelik ${ }^{1}$, Ötüken Senger ${ }^{2}$ \\ ${ }^{1}$ Atatürk University, Erzurum, Turkey \\ Ph.: +904422312122, e-mail: akemal.celik@atauni.edu.tr \\ ${ }^{2}$ Kafkas University, Kars, Turkey \\ Ph.: +904742251426, e-mail: otukensenger@gmail.com
}

\begin{abstract}
The main objective of this paper is to analyse user satisfaction with road, maritime, air, and rail transport services in Turkey using four distinctive ordered response models. The estimation results reveal that partial constrained generalized ordered logit model has the best model fit for road and maritime transport services. In addition, ordered logit and generalized ordered logit are the convenient models for air and rail transport services, respectively. Results also indicate that current residence (urban), gender (male), occupational sector (private), and education level (low educated) were the statistically significant variables that are more likely to increase user satisfaction. This paper is probably the first attempt to analyse user satisfaction with all transport services using comparative ordered response models. As a social indicator, the results of this study may provide a valuable evidence for future sustainable transport policies in Turkey.
\end{abstract}

Keywords: satisfaction, ordered logit model, generalized ordered logit model, heterogeneous choice model, partial constrained generalized ordered logit model, Turkey

\section{Introduction}

Sustainable transport is commonly adopted as finding a convenient balance between environmental, social and economic qualities by focusing on the positive and negative dimensions and externalities of traffic and transport for now or the near future. In this context, sustainable indicators are essentially required to examine possibilities and conditions for sustainable transport (Sted and Gifford, 2005). On the other hand, user satisfaction with public transit services can be briefly considered as the overall level of attainment of user's expectations which can be commonly measured as the percentage of the user expectations that have been fulfilled (Tyrinopoulos and Antoniou, 2008). User satisfaction surveys appear to be indispensable to determine which possible factors are more effective on the choice of public transit services by establishing differences based on varying socio-economic attributes (dell'Olio et al.,, 2010). In many aspects, user satisfaction surveys and ratings are highly recommended as one the most important economic (Litman, 2011) and social indicators (Litman, 2014) of sustainable transport, respectively. Since governments should encourage regular evaluation of the performance of the current transport system as an essential part of the sustainable planning process for assessing and benchmarking the effectiveness of the earlier policies, the crucial role of user satisfaction responses cannot be ignored (May, 2013).

In general, the use of transport services is steadily increasing in Turkey. As of August 2014, there were more than 18.5 million registered motor vehicles on Turkish highways (TurkStat, 2014a). Further, as of the late 2013, the average number of passengers who used road transport have reached to 1.4 billion (Turkish Ministry of Transport, Maritime Affairs and Communications 2013a). The total number of air traffic in Turkey was still gradually growing in 2013, which showed a significant $9.3 \%$ increase with respect to 2012. Despite a slight decrease of the daily traffic in Europe, Turkey had the highest traffic growth with an additional 200 flights per day to its network (Turkish General Directorate of State Airports Authority, 2014a). According to the latest statistics of the Turkish General Directorate of State Airports Authority (2014b), as of December 2013 and October 2014, there were totally more than 129 and 143 million passengers using air transport, respectively. However, the number of passengers who used railways decreased from nearly 70 million to 46.4 million between 2012 and 2013 despite a 4\% increase in high-speed railway transport use since 2009 (Turkish Ministry of Transport, Maritime Affairs and Communications, 2013b). Finally, the total number of passengers who used maritime transport services were 67,159 in 2007 (TurkStat, 2014b). 
The crucial relationship between the quality of transport services and sustainable transport has been extensively highlighted in the sustainable transport literature. Earlier studies (Paulley et al., 2006; Beirão and Sarfield Cabral, 2007; Eboli and Mazzulla, 2007; Feng and Hsieh, 2009; dell'Olio et al., 2010; Lai and Chen, 2011) found that quality level perceived by the user was the best indicator of user satisfaction. Another study (Shay and Khattak, 2010) argued that lack of user participation due to demographic factors may lead to policy-makers of transport employing the same traditional procedures for future planning and policies. Several past research also found significant evidence of the effect of demographic attributes on user satisfaction with transport services. A study conducted in Serbia (Filipović et al., 2009) found that retired passengers were more satisfied than student counterparts in terms of the transport service quality. Tyrinopoulos and Antoniou (2008) found that male respondents were more highly to value transport service frequency and accessibility than female counterparts. A very recent research carried out in Saudi Arabia (Al-Atawi and Saleh, 2014) indicated that male respondents, working in private company, military and health sector and level of education were significant indicators as a travel behaviour. A very recent study concerning high-speed railway service satisfaction in Turkey (Dölarslan, 2014) found that female, younger, low income and low educated users had more likely to have higher loyalty than other counterparts. Paulley et al. (2006) put forward the positive linkage between income level and the demand for public transport. Similar to these findings, Stathopoulos and Marcucci (2014) found income and age of the respondents were influencing factors of public transport service quality. In St-Louis et al. (2014)'s study, age and gender were also found as significant indicators of various users' preferences. Kroesen (2014) underlined the significant impact of age and residential environment on travel transition probabilities. Román et al. (2014) found that transport behaviour of urban and nonurban passengers differ in their perception of certain attributes and willing to pay. Diana (2012)'s survey pointed out the higher satisfaction of passengers living in small towns for local transit services.

Many previous work (Paulley et al., 2006; Beirão and Sarfield Cabral, 2007; Pantouvakis and Lymperopoulos, 2008; Tyrinopoulos and Antoniou, 2008; Filipović et al., 2009; dell'Olio et al., 2010; Too and Earl, 2010; Cirillo et al., 2011; Eboli and Mazzulla, 2011; Celik et al., 2013, 2014; Fiorio et al., 2013; Mokonyama and Venter, 2013; Redman et al., 2013; Al-Atawi and Saleh, 2014; Cascetta and Cartenì, 2014; De Oña et al., 2014; Garrido et al., 2014; Grujičić et al., 2014; Jain et al., 2014) also addressed the importance of physical and economic indicators such as functionality, comfort, cleanliness, courtesy of passengers or service providers, cheapness, security, etc. on user satisfaction with transport services.

The main objective of this paper is to determine key socio-economic factors affecting user satisfaction with transport services in Turkey. The remainder of the paper is outlined as follows. The next section includes conceptual background of the distinctive methodology used in the study and gives detailed information about the data collection. The results section introduces the estimation results with respect to four transport services in Turkey. The discussion section interprets the estimation results in parallel with past, present and future transport policy and makes suggestions for policy makers.

\section{Methods}

\subsection{Ordered response models}

Ordered categorical variables are frequently used in many social science applications. In principle, these type of variables denote the rank order of a particular attribute whilst such rankings do not necessarily represent the actual magnitudes on a substantive scale (Powers and Xie, 2000). When the outcomes are naturally ordered, the researcher should notice the fact that the dependent variable is considered as both discrete and ordinal. In other words, if the dependent variable has three categories, a linear regression would recognize the difference between category 3 and 2 identically to the difference between category 2 and 1 (Borooah, 2002).

The probability of an observed outcome such as $y=m$ for given values of $x$ 's designates to the region of the distribution where $y^{*}$ between $\tau_{m-1}$ and $\tau_{m}$ as

$$
\operatorname{Pr}(y=m \mid x)=\operatorname{Pr}\left(\tau_{m-1} \leq y^{*}<\tau_{m} \mid x\right)
$$

where $\tau$ 's are thresholds and $y^{*}$ is the latent variable. When $y^{*}$ is substituted with $x \beta+\varepsilon$ then Equation (1) can be rewritten as

$$
\operatorname{Pr}(y=m \mid x)=F\left(\tau_{m}-x \beta\right)-F\left(\tau_{m-1}-x \beta\right)
$$


where $F$ denotes the cumulative function for $\varepsilon$. Further, the ordered models can be developed as a nonlinear probability model without the idea of latent variables. For $m=1, J-1$, the odds that an outcome is then or equal to $m$ versus greater than $m$ given $x$ are as follows:

$\Omega_{\leq m \mid>m}(x) \equiv \frac{\operatorname{Pr}(y \leq m \mid x)}{\operatorname{Pr}(y>m \mid x)}$

For instance, assuming the logs of the odds is equal to

$\ln \Omega_{\leq m>m}(x)=\tau_{m}-x \beta$

the odds of $m \leq 2$ versus $m>2$ can be computed. For a simple three-category, the odds will be as the following (Long and Freese, 2001):

$$
\begin{aligned}
& \ln \frac{\operatorname{Pr}(y \leq 1 \mid x)}{\operatorname{Pr}(y>1 \mid x)}=\tau_{1}-\beta_{1} x_{1} \\
& \ln \frac{\operatorname{Pr}(y \leq 2 \mid x)}{\operatorname{Pr}(y>2 \mid x)}=\tau_{2}-\beta_{1} x_{1}
\end{aligned}
$$

Generalized ordered logit model can simply be defined as

$$
P\left(Y_{i}>j\right)=g\left(X \beta_{j}\right)=\frac{\exp \left(\alpha_{j}+X_{i} \beta_{j}\right)}{1+\left[\exp \left(\alpha_{j}+X_{i} \beta_{j}\right]\right.}, j=1,2, \ldots, M-1
$$

where $M$ is the number of categories of the ordinal dependent variable. It can be easily noticed that the parallel lines model differs from the standard generalized logit model except for the Betas that are the same for all categories. For instance, when there are four categories, first category $(J=1)$ is contrasted with category 2, 3, and 4 (Williams, 2006). Whilst the generalized model is frequently preferred, most researchers disregard the parallel lines assumption that is often violated (Fu, 1998). In that context, to overcome the limitations of parallel lines restrictions, partial proportional odds model is introduced as a special case of generalized logit model, whereas some of the Beta coefficients can differ. For instance, Equation (9) illustrates a partial proportional odds model which enables the Betas for $X 3$ to differ (Williams, 2006):

$$
P\left(Y_{i}>j\right)=g\left(X \beta_{j}\right)=\frac{\exp \left(\alpha_{j}+X 1_{i} \beta 1+X 2_{i} \beta 2+X 3_{i} \beta 3_{j}\right)}{1+\left[\exp \left(\alpha_{j}+X 1_{i} \beta 1+X 2_{i} \beta 2+X 3_{i} \beta 3_{j}\right)\right]}, j=1,2, \ldots, M-1
$$

Heterogeneous choice model provides the researchers to examine determinants of the conditional variance. For an ordered variable $y$ with $M$ categories, the full heterogeneous choice model can be written as

$$
P\left(y_{i}>m\right)=\operatorname{invlogit}\left\{\frac{\sum_{k} x_{i k} \beta_{k}-\kappa_{m}}{\exp \left(\sum_{j} z_{i j} \gamma_{j}\right)}\right\}=\operatorname{invlogit}\left(\frac{\sum_{k} x_{i k} \beta_{k}-\kappa_{m}}{\sigma_{i}}\right), m=1,2, \ldots, M-1
$$

where variance equation $\sigma_{i}$ can be defined as

$$
\sigma_{i}=\exp \left(\sum_{j} z_{i j} \gamma_{j}\right)
$$

For any given response, the full heterogeneous choice model in Equation (9) presents how the choice and variance equations are combined to put forward the probability (Williams, 2010).

Though regression parameters yield information about the sensitivity of a dependent variable regarding changes in several independent variables, in some circumstances, it may be more appropriate to 
measure these sensitivities in terms of percentages, where elasticities are also preferred. However, standard elasticity calculation is not considered as a valid measurement for indicator variables which were defined as dummies ( 1 for success and 0 for failure). For these types of variables a pseudo-elasticity measure given by

$$
E_{x_{k i}}^{P(i)}=\frac{\exp \left[\Delta\left(\beta_{i} x_{i}\right)\right] \sum_{\forall I} \exp \left(\beta_{k I} x_{k I}\right)}{\exp \left[\Delta\left(\beta_{i} x_{i}\right)\right] \sum_{\forall I} \exp \left(\beta_{k I} x_{k I}\right)+\sum_{\forall I \neq I_{n}} \exp \left(\beta_{k I} x_{k I}\right)}-1
$$

can be used, where $I_{n}$ denotes the set of alternate outcomes with $x_{k}$ in the function determining the outcome, and $I$ denotes the set of all possible outcomes. These elasticities capture the potential effect that a change in a variable determining the likelihood of alternative outcome $i$ has on the probability this outcome will be selected, which are also called as direct elasticities (Washington et al., 2003).

\subsection{Study Design, sample and data collection}

The present study utilized the data from 2011 and 2012 Life Satisfaction Survey (TurkStat, 2012, 2013) conducted by Turkish Statistical Institute among Turkish transport users after excluding the respondents who did not have any idea. The corresponding survey involved detailed questions about respondents' demographic characteristics and satisfaction levels regarding several transport services. The dependent variable of this study for the fitted models investigates the user satisfaction with road, air, marine, and rail transport services. The independent variable of this study had five ordinal hierarchical categories from 'very dissatisfied' to 'very satisfied'. For air transport services, two categories of dissatisfaction (namely, very dissatisfied and dissatisfied) and satisfaction (namely, satisfied and very satisfied) were merged because of relatively low responses. Due to the ordinal and discrete nature of the dependent variable, four distinctive ordered responses models were fitted, separately, such as ordered logit model, generalized ordered logit model, partial constrained generalized ordered model, and heterogeneous choice model. For simplicity, only seven independent variables were involved in the final model including current residence, gender, age, household size, marital status, educational level, and the occupational sector. Age of the respondent and household size were held as continuous variables and the rest of the independent variables were selected as dummy variables. The estimation results may also test the consistency of four models and provide the policy makers a benchmarking facility to decide on which model fits well.

\section{Estimation results}

\subsection{Descriptive statistics}

Table 1 presents descriptive statistics of both dependent and independent variables used for each transport services. As outlined in Table 1, a majority of respondents were satisfied or very satisfied with several transport services. For each transport services, most of the respondents were living in urban areas and similarly, most of them were married. The number of respondents regarding the gender variable were generally equal and for each transport services, nearly half of the respondents were literate or primary educated. For each transport services, more than $36 \%$ of the respondents were working in private sector. The average household size for four distinctive transport services varied between 3.50 and 3.78. Finally, the average age of the respondents was between 41.99 and 43.93 .

Table 1. Descriptive statistics of variables

\begin{tabular}{|c|c|c|c|c|c|c|c|c|}
\hline \multirow[t]{2}{*}{ Variable } & \multicolumn{2}{|c|}{$\begin{array}{c}\text { Road } \\
\text { transport }\end{array}$} & \multicolumn{2}{|c|}{$\begin{array}{l}\text { Maritime } \\
\text { Transport }\end{array}$} & \multicolumn{2}{|c|}{$\begin{array}{c}\text { Air } \\
\text { Transport } \\
\end{array}$} & \multicolumn{2}{|c|}{$\begin{array}{c}\text { Rail } \\
\text { Transport } \\
\end{array}$} \\
\hline & Frequency & Percent & Frequency & Percent & Frequency & Percent & Frequency & Percent \\
\hline \multicolumn{9}{|l|}{ User satisfaction } \\
\hline Very dissatisfied & 322 & 2.20 & 54 & 1.01 & \multirow[t]{2}{*}{$146^{\mathrm{b}}$} & \multirow[t]{2}{*}{2.87} & 90 & 1.48 \\
\hline Dissatisfied & 1,246 & 8.51 & 171 & 3.20 & & & 302 & 4.96 \\
\hline Somewhat satisfied & 1,487 & 10.15 & 468 & 8.77 & 350 & 6.87 & 523 & 8.60 \\
\hline Satisfied & 10.262 & 70.06 & 4,205 & 78.80 & \multirow{2}{*}{$4,598^{\mathrm{c}}$} & \multirow{2}{*}{90.26} & 4,613 & 75.83 \\
\hline Very satisfied $^{\mathrm{a}}$ & 1,331 & 9.09 & 438 & 8.21 & & & 555 & 9.12 \\
\hline \multicolumn{9}{|l|}{ Current residence } \\
\hline Urban & 10,839 & 74.00 & 4,714 & 88.34 & 4,389 & 86.16 & 4,660 & 76.61 \\
\hline Rural $^{\text {a }}$ & 3,809 & 26.00 & 622 & 11.66 & 705 & 13.84 & 1,423 & 23.39 \\
\hline
\end{tabular}




\begin{tabular}{|c|c|c|c|c|c|c|c|c|}
\hline \multicolumn{9}{|l|}{ Gender } \\
\hline Male & 6,683 & 45.62 & 2,690 & 50.41 & 2,710 & 53.20 & 2,846 & 46.79 \\
\hline Female $^{\mathrm{a}}$ & 7,965 & 54.38 & 2,646 & 49,59 & 2,384 & 46.80 & 3,327 & 53.21 \\
\hline \multicolumn{9}{|l|}{ Marital status } \\
\hline Single & 3,493 & 23.85 & 1,489 & 27.90 & 1,313 & 25.78 & 1,562 & 25.68 \\
\hline Married $^{\mathrm{a}}$ & 11,155 & 76.15 & 3,847 & 72.10 & 3,781 & 74.22 & 4,521 & 74.32 \\
\hline \multicolumn{9}{|l|}{ Educational level } \\
\hline Illiterate & 2,393 & 16.34 & 448 & 8.40 & 446 & 8.76 & 898 & 14.76 \\
\hline Literate/Primary & 7,617 & 52.00 & 2,635 & 49.38 & 2,273 & 44.62 & 3,112 & 51.16 \\
\hline Secondary & 2,714 & 18.53 & 1,264 & 23.69 & 1,208 & 23.71 & 1,179 & 19.38 \\
\hline Higher education $^{\mathrm{a}}$ & 1,924 & 13.13 & 989 & 18.53 & 1,167 & 22.91 & 894 & 14.70 \\
\hline \multicolumn{9}{|l|}{ Occupational sector } \\
\hline Public sector & 1,010 & 6.90 & 373 & 6.99 & 503 & 9.87 & 419 & 6.89 \\
\hline Private sector & 5,317 & 36.30 & 2,147 & 40.24 & 1,978 & 38.83 & 2,259 & 37.14 \\
\hline Retired & 1,739 & 11.87 & 720 & 13.49 & 746 & 14.64 & 765 & 12.58 \\
\hline Unemployed $^{\mathrm{a}}$ & 6,572 & 44.87 & 2,090 & 39.17 & 1,864 & 36.59 & 2,637 & 43.35 \\
\hline Average household size & \multicolumn{2}{|c|}{$3.78(1.87)^{\mathrm{d}}$} & \multicolumn{2}{|c|}{$3.55(1.55)^{\mathrm{d}}$} & \multicolumn{2}{|c|}{$3.50(1.57)^{\mathrm{d}}$} & \multicolumn{2}{|c|}{$3.74(1.83)^{\mathrm{d}}$} \\
\hline Average age & \multicolumn{2}{|c|}{$43.93(16.20)$} & \multicolumn{2}{|c|}{41.99 ( 15.37) } & \multicolumn{2}{|c|}{$43.10(15.45)$} & \multicolumn{2}{|c|}{$43.88(16.33)$} \\
\hline Number of observations & \multicolumn{2}{|c|}{14,648} & \multicolumn{2}{|c|}{5,336} & \multicolumn{2}{|c|}{5,094} & \multicolumn{2}{|c|}{6,083} \\
\hline
\end{tabular}

Notes: ${ }^{a}$ denotes base category; ${ }^{\mathrm{b}}$ denotes the sum of very dissatisfied and dissatisfied; ${ }^{\mathrm{c}}$ denotes the sum of satisfied and very satisfied; ${ }^{\mathrm{d}}$ standard deviations in parenthesis

\subsection{User satisfaction with road transport services}

Table 2 follows a similar design by Quddus et al. (2010) and introduces estimation results of ordered logit, generalized ordered logit, partial constrained generalized ordered logit and heterogeneous choice models for road transport services. A Wald test proposed by Brant (1990) was carried out to determine whether the parallel lines assumption was violated for the data being used in the analysis. Since a significant test statistic provided evidence that the corresponding assumption has been violated (Quddus et al., 2010), alternative three ordered response models were fitted by two user-written Stata routines (Williams, 2006, 2010). A comparison among four distinctive models regarding Akaike Information Criterion (AIC) values confirmed that partial constrained generalized ordered model was the best model with relatively lower AIC value.

The interpretation of the estimation models were accomplished using average direct pseudoelasticities in Table 3 which was highly recommended for dummy variables. Whilst marginal effects of two continuous variables were calculated, they were presented in any tables because of their negligible effects on any satisfaction levels. Additionally, significant effects of dummy variables under $0.1 \%$ were also ignored. Since partial constrained generalized logit model had the best fit, the interpretation of the average direct pseudo-elasticies was done considering this model. Accordingly, urban users were $43.4 \%$ less likely to be very dissatisfied with road transport services than rural counterparts. On the other hand, single respondents were more likely to be very dissatisfied with these services than married respondents. The average pseudo-elasticity value for illiterate and literate/primary educated respondents confirms that the probability of being very dissatisfied for these groups decrease by $12.8 \%$ and $36.8 \%$, respectively. In contrast, secondary educated respondents were slightly $(6.0 \%)$ more likely to be very dissatisfied than higher educated respondents. Respondents who were working in public and private sectors were more likely ( $2.7 \%$ and $2.9 \%$, respectively) to be very satisfied with road transport services than unemployed users.

The estimation results indicated that the probability of urban users' dissatisfaction decreased by $14.4 \%$ than rural users. Single users were more dissatisfied with road transport services than married counterparts. Regarding education level, both illiterate and literate/primary educated users were less likely $(8.9 \%$ and $26.8 \%)$ to be dissatisfied than higher educated road transport users. Public and private users were more likely to be dissatisfied with road transport services than unemployed users. Similar estimation results were obtained for 'somewhat satisfied' level. Accordingly, single respondents were more likely to be somewhat satisfied with road transport services than married respondents. All education levels were less likely to be somewhat satisfied with road transport services than higher educated counterparts. The probability of somewhat satisfaction with road transport services for private users slightly increase by $2.1 \%$. The analysis results suggested that urban or female users were more likely to be satisfied than rural or male respondents. In addition, all education levels were more likely to be satisfied with road transport services than higher educated counterparts. Male or married users were more likely to be very satisfied with road transport services than female or single users. Finally, road transport service users working in private sector were more likely to be very satisfied than unemployed users. 
Table 2. Results for road transport user satisfaction

\begin{tabular}{|c|c|c|c|c|c|c|c|c|c|c|c|c|}
\hline \multirow{2}{*}{\multicolumn{2}{|c|}{ User satisfaction with road transport services }} & \multirow{2}{*}{\begin{tabular}{|c|}
$\begin{array}{c}\text { Ordered } \\
\text { Logit }\end{array}$ \\
Coefficient \\
\end{tabular}} & \multirow{2}{*}{$\begin{array}{c}\text { Heterogeneous } \\
\text { Choice Model } \\
\text { Coefficient }\end{array}$} & \multicolumn{4}{|c|}{$\begin{array}{c}\text { Generalized } \\
\text { Ordered Logit }\end{array}$} & \multicolumn{5}{|c|}{$\begin{array}{c}\text { Partial Constrained } \\
\text { Generalized Ordered Logit }\end{array}$} \\
\hline & & & & $\begin{array}{c}\text { Threshold } \\
1 \text { and } 2 \\
\end{array}$ & \begin{tabular}{|l|} 
Threshold \\
2 and 3 \\
\end{tabular} & \begin{tabular}{|c|} 
Threshold \\
3 and 4
\end{tabular} & $\begin{array}{l}\text { Threshold } \\
4 \text { and } 5\end{array}$ & $\begin{array}{c}\text { Coeff. not } \\
\text { varying }\end{array}$ & $\begin{array}{r}\text { Threshold } \\
1 \text { and } 2 \\
\end{array}$ & \begin{tabular}{|l|} 
Threshold \\
2 and 3
\end{tabular} & \begin{tabular}{|c} 
Threshold \\
3 and 4 \\
\end{tabular} & $\begin{array}{c}\text { Threshold } \\
4 \text { and } 5\end{array}$ \\
\hline \multirow{17}{*}{$\begin{array}{l}\text { Factors affecting } \\
\text { the ordinal } \\
\text { dependent variable }\end{array}$} & Current residence & & & & & & & & & & & \\
\hline & Urban & $0.0923^{\mathrm{b}}$ & $0.0938^{\mathrm{b}}$ & $0.5856^{\mathrm{a}}$ & $0.2995^{\mathrm{a}}$ & $0.1692^{\mathrm{a}}$ & $-0.1243^{\mathrm{c}}$ & - & $0.5974^{\mathrm{a}}$ & $0.2940^{\mathrm{a}}$ & $0.1612^{\mathrm{a}}$ & -0.1096 \\
\hline & Household size & $-0.0356^{\mathrm{a}}$ & $-0.0359^{\mathrm{a}}$ & $-0.0530^{\mathrm{c}}$ & -0.0256 & $-0.0035^{\mathrm{a}}$ & $-0.0505^{\mathrm{a}}$ & $-0.0385^{\mathrm{a}}$ & - & - & - & - \\
\hline & Gender & & & & & & & & & & & \\
\hline & Male & $0.1257^{\mathrm{a}}$ & $0.1293^{\mathrm{a}}$ & 0.0668 & 0.0167 & 0.0062 & $0.4227^{\mathrm{a}}$ & - & 0.0552 & -0.0021 & 0.0195 & $0.4003^{\mathrm{a}}$ \\
\hline & Age & $0.0092^{\mathrm{a}}$ & $0.0095^{\mathrm{a}}$ & $0.0256^{\mathrm{a}}$ & 0.0171 & $0.0121^{\mathrm{a}}$ & 0.0019 & - & $0.0291^{\mathrm{a}}$ & $0.0153^{\mathrm{a}}$ & $0.0111^{\mathrm{a}}$ & $0.0040^{\mathrm{c}}$ \\
\hline & Marital status & & & & & & & & & & & \\
\hline & Single & $-0.2394^{\mathrm{a}}$ & $-0.2434^{\mathrm{a}}$ & $-0.3250^{\mathrm{b}}$ & $-0.1870^{\mathrm{a}}$ & $-0.1844^{\mathrm{a}}$ & $-0.2951^{\mathrm{a}}$ & $-0.2158^{\mathrm{a}}$ & - & - & - & - \\
\hline & Educational level & & & & & & & & & & & \\
\hline & Illiterate & $0.5052^{\mathrm{a}}$ & $0.5226^{\mathrm{a}}$ & $0.8374^{\mathrm{a}}$ & $0.5911^{\mathrm{a}}$ & $0.7301^{\mathrm{a}}$ & -0.1307 & - & $0.7968^{\mathrm{a}}$ & $0.6547^{\mathrm{a}}$ & $0.7384^{\mathrm{a}}$ & -0.1586 \\
\hline & Literate/Primary & $0.5870^{\mathrm{a}}$ & $0.6052^{\mathrm{a}}$ & $0.7104^{\mathrm{a}}$ & $0.5825^{\mathrm{a}}$ & $0.8000^{\mathrm{a}}$ & 0.1254 & - & $0.7211^{\mathrm{a}}$ & $0.6113^{\mathrm{a}}$ & $0.7860^{\mathrm{a}}$ & 0.0122 \\
\hline & Secondary & $0.2594^{\mathrm{a}}$ & $0.2662^{\mathrm{a}}$ & $0.3306^{\mathrm{c}}$ & $0.1719^{\mathrm{c}}$ & $0.3290^{\mathrm{a}}$ & 0.0174 & - & $0.3306^{\mathrm{c}}$ & $0.1878^{\mathrm{b}}$ & $0.3251^{\mathrm{a}}$ & 0.0179 \\
\hline & Occupational sector & & & & & & & & & & & \\
\hline & Public sector & -0.0965 & -0.1022 & $-0.4246^{\mathrm{b}}$ & $-0.3321^{\mathrm{a}}$ & -0.0875 & -0.0121 & - & $-0.3988^{b}$ & $-0.2938^{\mathrm{a}}$ & -0.1093 & 0.0271 \\
\hline & Private sector & -0.0712 & -0.0574 & -0.1101 & $-0.1226^{\mathrm{c}}$ & -0.0451 & $-0.1536^{\mathrm{c}}$ & -0.0826 & - & - & - & - \\
\hline & Retired & 0.0165 & 0.0228 & 0.2436 & -0.1280 & -0.0358 & 0.0588 & 0.0029 & - & - & - & - \\
\hline & Constant & - & - & $2.1782^{\mathrm{a}}$ & $1.0139^{\mathrm{a}}$ & $0.3361^{z}$ & -2.1994 & - & $1.9505^{\mathrm{a}}$ & $1.1016^{\mathrm{a}}$ & $0.4109^{\mathrm{a}}$ & $-2.3692^{\mathrm{a}}$ \\
\hline \multirow{7}{*}{$\begin{array}{l}\text { Factors affecting } \\
\text { the error variance }\end{array}$} & Current residence & & & & & & & & & & & \\
\hline & Urban & - & 0.0029 & - & - & - & - & - & - & - & - & - \\
\hline & Marital status & & & & & & & & & & & \\
\hline & Single & - & 0.0165 & - & - & - & - & - & - & - & - & - \\
\hline & Occupational sector & & & & & & & & & & & \\
\hline & Public sector & - & $0.0476^{\mathrm{a}}$ & - & - & - & - & - & - & - & - & - \\
\hline & Private sector & - & 0.0126 & - & - & - & - & - & - & - & - & - \\
\hline \multirow[t]{5}{*}{ Statistics } & Cut point 1 & -3.1056 & $-3.2116^{\mathrm{a}}$ & - & - & - & - & - & - & - & - & - \\
\hline & Cut point 2 & -1.4177 & $-1.4595^{\mathrm{a}}$ & - & - & - & - & - & - & - & - & - \\
\hline & \begin{tabular}{|l|} 
Cut point 3 \\
Cut point 4 \\
\end{tabular} & $\begin{array}{r}-0.6152 \\
3.0964 \\
\end{array}$ & $\begin{array}{r}-0.6277^{\mathrm{a}} \\
3.2079^{\mathrm{a}} \\
\end{array}$ & - & - & - & - & - & - & - & - & - \\
\hline & \begin{tabular}{|l|} 
Log-likelihood \\
\end{tabular} & $-14,366.2$ & $-14,361.9$ & & $-14,427.6$ & & & & & $-14,253.6$ & & \\
\hline & AIC & $28,762.49$ & $28,784.19$ & & $28,591.28$ & & & & & $28,579.29$ & & \\
\hline
\end{tabular}

Notes: a significant at $99 \%$; ${ }^{b}$ significant at $95 \%$; ${ }^{\text {c }}$ significant at $90 \%$ 
Table 3. Average direct pseudo-elasticities for road transport user satisfaction

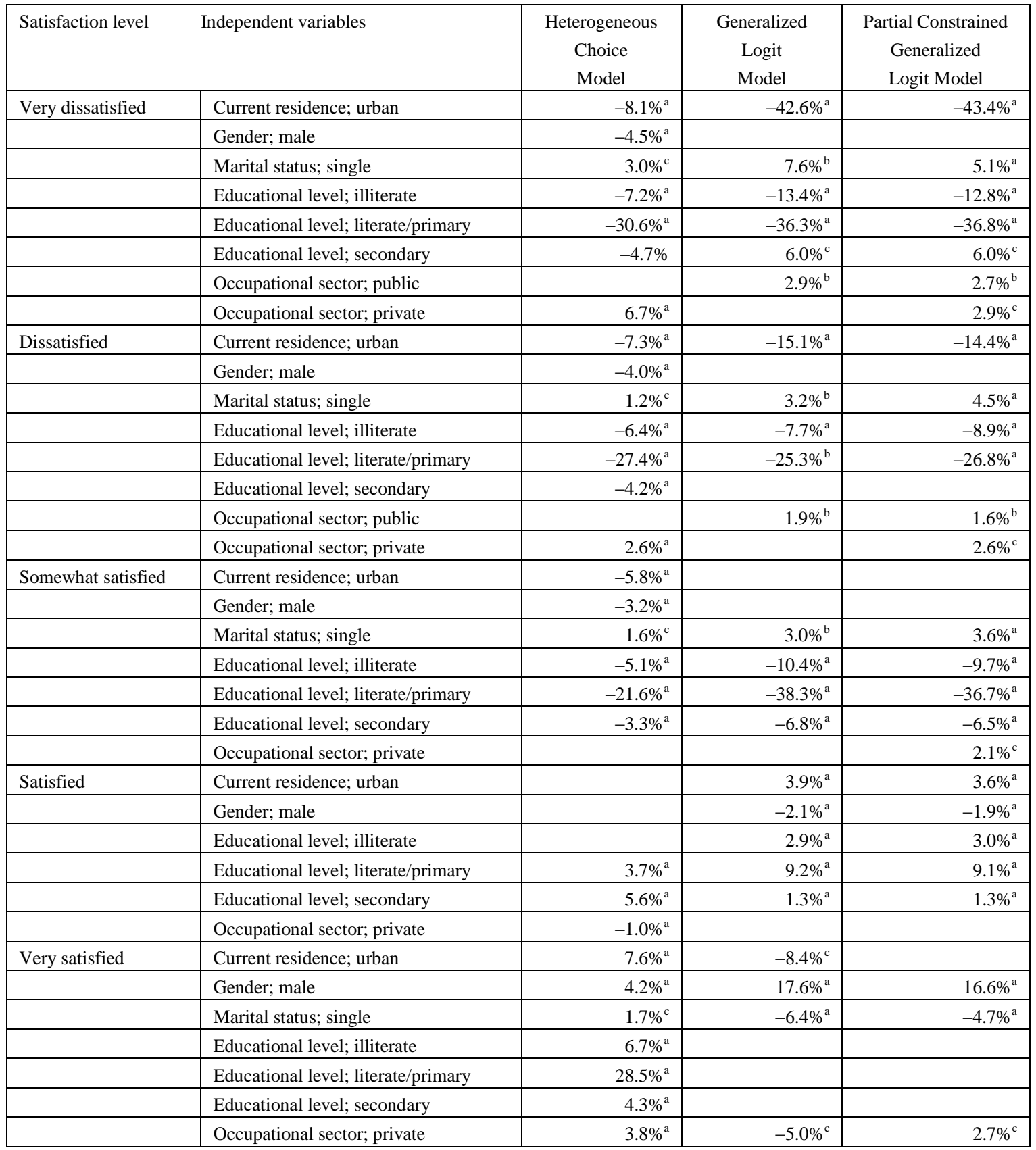

Notes: ${ }^{\text {a }}$ significant at $99 \% ;{ }^{b}$ significant at $95 \% ;{ }^{c}$ significant at $90 \%$

\subsection{User satisfaction with maritime transport services}

Table 4 indicates estimation results of four ordered response models for maritime transport services. Similar to road transport services, ordered logit models did violate the parallel lines assumption and alternative ordered response models were employed. The partial constrained generalized logit model had the lower AIC value and thus the best model choice for maritime transport services. The averagepseudo elasticity values (Table 5) for this model were interpreted. Accordingly, rural maritime transport users were more likely to be very dissatisfied or dissatisfied than urban users. Female respondents were less likely to be dissatisfied with maritime transport services than male counterparts. In contrast, higher educated maritime transport users were more likely to be dissatisfied than illiterate and literate/primary educated users. Respondents working in public sector were more likely to be dissatisfied than unemployed counterparts. Higher educated respondents were more likely to be somewhat satisfied than all other education levels, while unemployed respondents were more likely to be somewhat satisfied than private respondents. 
Table 4. Results for marine transport user satisfaction

\begin{tabular}{|c|c|c|c|c|c|c|c|c|c|c|c|c|}
\hline \multirow{2}{*}{\multicolumn{2}{|c|}{ User satisfaction with marine transport services }} & \multirow{3}{*}{\begin{tabular}{|c|}
$\begin{array}{c}\text { Ordered } \\
\text { Logit }\end{array}$ \\
Coefficient
\end{tabular}} & \multirow{3}{*}{$\begin{array}{c}\begin{array}{c}\text { Heterogeneous } \\
\text { Choice Model }\end{array} \\
\text { Coefficient }\end{array}$} & \multirow{2}{*}{\multicolumn{4}{|c|}{$\begin{array}{l}\text { Generalized } \\
\text { Ordered Logit }\end{array}$}} & \multirow{2}{*}{\multicolumn{5}{|c|}{$\begin{array}{c}\text { Partial Constrained } \\
\text { Generalized Ordered Logit }\end{array}$}} \\
\hline & & & & & & & & & & & & \\
\hline & & & & \multirow[t]{2}{*}{$\begin{array}{c}\text { Threshold } \\
1 \text { and } 2\end{array}$} & \multirow[t]{2}{*}{$\begin{array}{l}\text { Threshold } \\
2 \text { and } 3\end{array}$} & \multirow[t]{2}{*}{$\begin{array}{l}\text { Threshold } \\
3 \text { and } 4\end{array}$} & \multirow[t]{2}{*}{$\begin{array}{l}\text { Threshold } \\
4 \text { and } 5\end{array}$} & \multirow[t]{2}{*}{$\begin{array}{c}\text { Coeff. not } \\
\text { varying }\end{array}$} & \multirow[t]{2}{*}{$\begin{array}{r}\text { Threshold } \\
1 \text { and } 2\end{array}$} & \multirow[t]{2}{*}{$\begin{array}{l}\text { Threshold } \\
2 \text { and } 3\end{array}$} & \multirow{2}{*}{\multicolumn{2}{|c|}{\begin{tabular}{|l} 
Threshold Threshold \\
3 and $4 \quad 4$ and 5
\end{tabular}}} \\
\hline \multirow{17}{*}{$\begin{array}{l}\text { Factors affecting } \\
\text { the ordinal } \\
\text { dependent variable }\end{array}$} & Current residence & & & & & & & & & & & \\
\hline & Urban & 0.0224 & 0.0290 & $0.6238^{\mathrm{c}}$ & $0.6524^{\mathrm{a}}$ & 0.1998 & $-0.2442^{\mathrm{c}}$ & - & $0.6350^{\mathrm{c}}$ & $0.5698^{\mathrm{a}}$ & 0.1907 & -0.2261 \\
\hline & Household size & $-0.0672^{\mathrm{a}}$ & -0.0674 & $-0.1725^{\mathrm{c}}$ & -0.0397 & $-0.0676^{\mathrm{b}}$ & $-0.0668^{\mathrm{c}}$ & $-0.0661^{\mathrm{a}}$ & - & - & - & - \\
\hline & Gender & & & & & & & & & & & \\
\hline & Male & -0.0108 & -0.0128 & -0.2583 & $-0.2645^{\mathrm{c}}$ & $-0.2058^{\mathrm{b}}$ & $0.3321^{\mathrm{a}}$ & - & -0.4000 & $-0.3173^{\mathrm{b}}$ & $-0.2235^{\mathrm{b}}$ & 0.0020 \\
\hline & Age & 0.0038 & 0.0040 & -0.0165 & 0.0007 & 0.0059 & 0.0019 & - & -0.0150 & -0.0034 & $0.0058^{\mathrm{c}}$ & 0.3692 \\
\hline & Marital status & & & & & & & & & & & \\
\hline & \begin{tabular}{|l} 
Single \\
\end{tabular} & -0.0955 & -0.0228 & -0.2193 & 0.1125 & -0.1099 & $-0.0848^{\mathrm{a}}$ & -0.0941 & - & - & - & - \\
\hline & Educational level & & & & & & & & & & & \\
\hline & Illiterate & $0.6208^{\mathrm{a}}$ & $0.6193^{\mathrm{a}}$ & 0.5097 & $0.7968^{b}$ & $0.6322^{\mathrm{a}}$ & $0.3998^{\mathrm{c}}$ & $0.5390^{\mathrm{a}}$ & - & - & - & - \\
\hline & Literate/Primary & $0.5111^{\mathrm{a}}$ & $0.5131^{\mathrm{a}}$ & 0.3878 & $0.9685^{\mathrm{a}}$ & $0.7069^{\mathrm{a}}$ & 0.0753 & - & 0.4846 & $0.8850^{\mathrm{a}}$ & $0.6864^{\mathrm{a}}$ & 0.1375 \\
\hline & Secondary & $0.2682^{\mathrm{b}}$ & $0.2731^{\mathrm{b}}$ & 0.5015 & $0.3870^{\mathrm{b}}$ & $0.4342^{\mathrm{a}}$ & -0.1377 & - & 0.4719 & $0.3034^{\mathrm{c}}$ & $0.4141^{\mathrm{a}}$ & -0.0794 \\
\hline & Occupational sector & & & & & & & & & & & \\
\hline & Public sector & -0.0731 & -0.0802 & -0.4534 & $-0.4245^{\mathrm{a}}$ & 0.0292 & -0.1972 & - & -0.0884 & $-0.4845^{\mathrm{b}}$ & 0.0307 & -0.1856 \\
\hline & Private sector & 0.0914 & 0.0925 & -0.2511 & -0.0340 & $0.2224^{\mathrm{b}}$ & -0.1338 & - & 0.1164 & 0.0432 & $0.2335^{b}$ & -0.1592 \\
\hline & Retired & -0.0345 & -0.0228 & -0.7514 & -0.2709 & -0.1223 & 0.0316 & -0.0636 & - & - & - & - \\
\hline & Constant & - & - & $5.5236^{\mathrm{a}}$ & $2.2887^{\mathrm{a}}$ & $1.3132^{\mathrm{a}}$ & -2.1882 & - & $4.7667^{\mathrm{a}}$ & $2.7566^{\mathrm{a}}$ & $1.3318^{\mathrm{a}}$ & -2.2651 \\
\hline \multirow{7}{*}{$\begin{array}{l}\text { Factors affecting } \\
\text { the error variance }\end{array}$} & Household size & - & -0.0171 & - & - & - & - & - & - & - & - & - \\
\hline & Marital status & & & & & & & & & & & \\
\hline & Single & - & 0.0104 & - & - & - & - & - & - & - & - & - \\
\hline & Education level & & & & & & & & & & & \\
\hline & Illiterate & - & 0.0379 & - & - & - & - & - & - & - & - & - \\
\hline & Occupational sector & & & & & & & & & & & \\
\hline & Retired & - & $0.0890^{\mathrm{b}}$ & - & - & - & - & - & - & - & - & - \\
\hline \multirow[t]{6}{*}{ Statistics } & Cut point 1 & -4.3100 & $-4.3534^{\mathrm{a}}$ & - & - & - & - & - & - & - & - & - \\
\hline & Cut point 2 & -2.8469 & $-2.8673^{\mathrm{a}}$ & - & - & - & - & - & - & - & - & - \\
\hline & \begin{tabular}{|l|} 
Cut point 3 \\
Cut point 4
\end{tabular} & $\begin{array}{r}-1.6183 \\
2.7480\end{array}$ & $\begin{array}{r}-1.6236^{\mathrm{a}} \\
2.7980^{\mathrm{a}}\end{array}$ & - & - & - & - & - & - & $\overline{-}$ & - & $\begin{array}{l}- \\
-\end{array}$ \\
\hline & \begin{tabular}{|l} 
Log-likelihood \\
\end{tabular} & $-4,043.02$ & $-4,039.93$ & & $-4,002.80$ & & & & & $-4,007.98$ & & \\
\hline & LR Chi-square (df) & $58.00(11)$ & $64.2(15)$ & & $138.4(44)$ & & & & & $128.1(32)$ & & \\
\hline & AIC & $8,116.04$ & $8,117.87$ & & $8,101.61$ & & & & & \begin{tabular}{|l|}
$8,087.96$ \\
\end{tabular} & & \\
\hline
\end{tabular}

Notes: ${ }^{\text {a }}$ significant at $99 \% ;{ }^{\text {b }}$ significant at $95 \% ;{ }^{c}$ significant at $90 \%$ 
The estimation results for maritime transport services put forward that urban respondents were more likely to be satisfied than rural respondents. With respect to gender, female maritime transport users were more likely to be satisfied than male counterparts. Higher educated respondents were less likely to be satisfied than all other education levels. Respondents working in private sector were more likely to be satisfied than unemployed respondents. Finally, male or illiterate respondents were more likely to be very satisfied with maritime transport services than female or higher educated counterparts, respectively.

Table 5. Average direct pseudo-elasticities for maritime transport user satisfaction

\begin{tabular}{|c|c|c|c|c|}
\hline Satisfaction level & ndependent variables & $\begin{array}{c}\text { Heterogeneous } \\
\text { Choice } \\
\text { Model }\end{array}$ & $\begin{array}{l}\text { Generalized } \\
\text { Logit } \\
\text { Model }\end{array}$ & $\begin{array}{c}\text { Partial Constrained } \\
\text { Generalized } \\
\text { Logit Model }\end{array}$ \\
\hline \multirow{3}{*}{ Very dissatisfied } & Educational level; illiterate & & & $-4.5 \%^{\mathrm{a}}$ \\
\hline & Educational level; literate/primary & $-23.0 \%{ }^{\mathrm{a}}$ & & \\
\hline & Occupational sector; retired & $5.7 \%^{\mathrm{b}}$ & & \\
\hline \multirow[t]{5}{*}{ Dissatisfied } & Current residence; urban & & $-55.7 \%^{\mathrm{a}}$ & $-46.1 \%^{b}$ \\
\hline & Gender; male & & & $13.9 \%^{\mathrm{c}}$ \\
\hline & Educational level; illiterate & $-32.5 \%^{\mathrm{b}}$ & $-7.1 \%^{\mathrm{b}}$ & $-4.3 \%^{\mathrm{a}}$ \\
\hline & Occupational sector; public & & & $4.1 \%^{\mathrm{b}}$ \\
\hline & Occupational sector; retired & $3.2 \%{ }^{\mathrm{b}}$ & & \\
\hline \multirow[t]{6}{*}{ Somewhat satisfied } & Marital status; single & & $5.1 \%^{\mathrm{c}}$ & \\
\hline & Educational level; illiterate & $-32.9 \%^{\mathrm{a}}$ & $-3.9 \%^{\mathrm{b}}$ & $-3.8 \%^{\mathrm{a}}$ \\
\hline & Educational level; literate/primary & $-19.3 \%^{\mathrm{a}}$ & $-23.9 \%^{\mathrm{a}}$ & $-24.2 \%^{\mathrm{a}}$ \\
\hline & Educational level; secondary & $-4.9 \%{ }^{b}$ & $-9.1 \%^{\mathrm{a}}$ & $-9.3 \%^{\mathrm{a}}$ \\
\hline & Occupational sector; private & & $-10.6 \%^{\mathrm{b}}$ & $-11.1 \%^{\mathrm{c}}$ \\
\hline & Occupational sector; retired & $1.3 \%$ & & \\
\hline Satisfied & Occupational sector; private & & $1.7 \%^{\mathrm{b}}$ & $1.9 \%^{\mathrm{c}}$ \\
\hline \multirow[t]{6}{*}{ Very satisfied } & Current residence; urban & & $-19.8 \%^{\mathrm{c}}$ & \\
\hline & Gender; male & & $15.4 \%^{\mathrm{a}}$ & $17.1 \%^{\mathrm{a}}$ \\
\hline & Educational level; illiterate & $4.9 \%{ }^{\mathrm{a}}$ & $3.1 \%{ }^{\mathrm{c}}$ & $4.2 \%{ }^{\mathrm{a}}$ \\
\hline & Educational level; literate/primary & $21.3 \%^{\mathrm{a}}$ & & \\
\hline & Educational level; secondary & $5.5 \%^{\mathrm{b}}$ & & \\
\hline & Occupational sector; retired & $2.8 \%^{\mathrm{a}}$ & & \\
\hline
\end{tabular}

Notes: ${ }^{a}$ significant at $99 \% ;{ }^{\text {b }}$ significant at $95 \% ;^{\mathrm{c}}$ significant at $90 \%$

\subsection{User satisfaction with air transport services}

Table 6 indicates estimation results for air transport user satisfaction. An insignificant test statistic obtained from the Wald test by Brant (1990) confirmed that the ordered logit model did not violate the parallel lines assumption and it was the best specified model for air transport service user satisfaction. Again, the average pseudo-elasticities were preferred for the interpretation. 
Table 6. Estimation results for air transport user satisfaction

\begin{tabular}{|c|c|c|c|}
\hline \multicolumn{2}{|c|}{ User satisfaction with air transport services } & \multicolumn{2}{|c|}{$\begin{array}{l}\text { Ordered Logit } \\
\text { Model }\end{array}$} \\
\hline & & Coefficient & Z-statistic \\
\hline \multirow{16}{*}{$\begin{array}{l}\text { Factors affecting } \\
\text { the ordinal } \\
\text { dependent variable }\end{array}$} & Current residence & & \\
\hline & Urban & 0.1905 & 1.38 \\
\hline & Household size & $-0.0698^{\mathrm{b}}$ & -2.21 \\
\hline & Gender & & \\
\hline & Male & 0.1527 & 1.37 \\
\hline & Age & $0.0110^{\mathrm{b}}$ & 2.48 \\
\hline & Marital status & & \\
\hline & Single & 0.0633 & 0.54 \\
\hline & Educational level & & \\
\hline & Illiterate & $0.4636^{\mathrm{b}}$ & 1.97 \\
\hline & Literate/Primary & $0.3870^{\mathrm{a}}$ & 2.82 \\
\hline & Secondary & 0.2304 & 1.64 \\
\hline & Occupational sector & & \\
\hline & Public sector & -0.1442 & -0.78 \\
\hline & Private sector & 0.0824 & 0.64 \\
\hline & Retired & -0.0936 & -0.49 \\
\hline \multirow[t]{6}{*}{ Statistics } & Cut point 1 & -2.7989 & \\
\hline & Cut point 2 & -1.4966 & \\
\hline & Number of observations & 5,094 & \\
\hline & Log-likelihood & $-1,908.32$ & \\
\hline & LR Chi-square (df) & $37.18(11)$ & \\
\hline & AIC & $3,842.641$ & \\
\hline
\end{tabular}

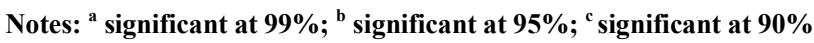

As shown in Table 7, education level was the only statistically significant indicator for all satisfaction levels. Accordingly, higher educated users of air transport services were more likely to be very dissatisfied and dissatisfied than illiterate and literate/primary counterparts in terms of education levels. Similarly, higher educated respondents were more likely to somewhat satisfied than less educated users including illiterate and literate/primary. Not surprisingly, illiterate and literate/primary educated air transport service users were more likely to be satisfied or very satisfied than higher educated counterparts.

Table 7. Average direct pseudo-elasticities for air transport user satisfaction

\begin{tabular}{|l|l|c|}
\hline \multicolumn{2}{|l|}{ Pseudo-elasticity for air transport user satisfaction } & Ordered Logit Model \\
\hline Very dissatisfied/dissatisfied & Educational level; illiterate & $-3.9 \%^{\mathrm{b}}$ \\
\hline & Educational level; literate/primary & $-16.8 \%^{\mathrm{a}}$ \\
\hline Somewhat satisfied & Educational level; illiterate & $-3.6 \%^{\mathrm{b}}$ \\
\hline & Educational level; literate/primary & $-15.2 \%^{\mathrm{a}}$ \\
\hline Satisfied/very satisfied & Educational level; illiterate & $3.8 \%^{\mathrm{b}}$ \\
\hline & Educational level; literate/primary & $1.6 \%^{\mathrm{a}}$ \\
\hline
\end{tabular}

Notes: ${ }^{a}$ significant at $99 \% ;{ }^{b}$ significant at $95 \%$; ${ }^{\text {c }}$ significant at $90 \%$

\subsection{User satisfaction with rail transport services}

The generalized logit model was the only statistically significant alternative of the ordered logit model that violated the parallel lines assumption for rail transport services in Table 8. Apart from all other fitted models in this study, this model was only statistically significant at $90 \%$. Whilst heterogeneous choice and partial constrained generalized logit model trials were performed for rail transport service data, none of these models satisfied statistically significance at any levels. The generalized ordered logit model was consistently interpreted with respect to the average direct pseudo-elasticities in Table 9. 
Table 8. Estimation results for rail transport user satisfaction

\begin{tabular}{|l|l|r|r|}
\hline \multirow{2}{*}{\begin{tabular}{l} 
User satisfaction with rail transport services \\
\multirow{3}{*}{$\begin{array}{l}\text { Factors affecting } \\
\text { the ordinal } \\
\text { dependent variable }\end{array}$}
\end{tabular}} & \multicolumn{2}{c|}{$\begin{array}{c}\text { Generalized } \\
\text { Ordered Logit }\end{array}$} \\
\cline { 2 - 4 } & Threshold 1 and 2 & & Z-statistic \\
\cline { 2 - 4 } & Household size & $-0.0963^{\mathrm{c}}$ & -1.92 \\
\cline { 2 - 4 } & Constant & $3.7814^{\mathrm{a}}$ & 6.45 \\
\hline & Threshold 2 and 3 & $0.4465^{\mathrm{c}}$ & 1.68 \\
\hline & Occupational sector; public sector & $0.2430^{\mathrm{c}}$ & 1.70 \\
\hline & Occupational sector; private sector & $2.5524^{\mathrm{a}}$ & 7.73 \\
\hline & Constant & & -1.95 \\
\hline & Threshold 3 and 4 & $-0.0057^{\mathrm{c}}$ & -2.26 \\
\hline & Age & $-0.1894^{\mathrm{b}}$ & 1.67 \\
\hline & Marital status; single & $0.2322^{\mathrm{c}}$ & \\
\hline & Occupational sector; retired & $2.0951^{\mathrm{a}}$ & 9.35 \\
\hline & Constant & & \\
\hline & Threshold 4 and 5 & $0.3446^{\mathrm{a}}$ & \\
\hline & Current residence; urban & $0.2332^{\mathrm{b}}$ & 2.90 \\
\hline & Gender; male & $-2.8740^{\mathrm{a}}$ & -10.3 \\
\hline & Constant & $-5,145.5$ \\
\hline
\end{tabular}

Notes: ${ }^{a}$ significant at $99 \% ;{ }^{b}$ significant at $95 \% ;{ }^{c}$ significant at $90 \%$

As shown in Table 9, satisfaction levels of 'very dissatisfied' and 'somewhat satisfied' did not produce any statistically significant indicators. Furthermore; current residence, gender and occupational sector were potential indicators of rail transport user satisfaction. Users of public sectors were less likely to be dissatisfied than unemployed users of rail transport services. Urban or male users were less likely to be satisfied than rural or female rail transport users. On the other hand, urban and male users were more likely $(24.0 \%$ and $9.9 \%$, respectively) to be very satisfied with rail transport services than rural and female counterparts, respectively.

Table 9. Average direct pseudo-elasticities for rail transport user satisfaction

\begin{tabular}{|l|l|c|}
\hline \multicolumn{2}{|l|}{ Pseudo-elasticity for rail transport user satisfaction } & $\begin{array}{c}\text { Generalized Logit } \\
\text { Model }\end{array}$ \\
\hline Dissatisfied & Occupational sector; public & $-4.1 \%^{\mathrm{b}}$ \\
\hline Satisfied & Current residence; urban & $-3.9 \%^{\mathrm{a}}$ \\
\hline & Gender; male & $-2.0 \%^{\mathrm{b}}$ \\
\hline Very satisfied & Current residence; urban & $24.0 \%^{\mathrm{a}}$ \\
\hline & Gender; male & $9.9 \%^{\mathrm{b}}$ \\
\hline
\end{tabular}

Notes: ${ }^{a}$ significant at $99 \%$; ${ }^{b}$ significant at $95 \%$

\section{Concluding remarks}

Sustainable transport is mainly examined by evaluating the sustainability of current transport systems that implies the necessity of considering a broader range of sustainability indicators (Steg and Gifford 2005). Since many transport policy decisions are frequently accomplished in terms of service quality, the determination of useful performance measures emerges as a crucial issue in the sustainable transport planning field (Gulhan et al., 2014). Further, the use of service quality indicators and related measurement methods enables the translation of customer expectations into measurable quality parameters (Cascetta and Cartenì, 2014). User satisfaction with distinctive transport services are frequently adopted as one of the most important social indicators of further sustainable transport policies. However, sustainable transport research seems to be somewhat neglected in Turkey except for some previous work (e.g., Haldenbilen, 2003; Cirit ,2014). The present study aims to fill this crucial gap in the existing literature by taking account the user satisfaction with all transport services. This study is probably the first attempt to analyse four distinctive transport services using four different ordered response models. Additionally, regarding the important role of user satisfaction surveys on the success of sustainable transport, the estimation results of this study may be considered as a valuable social indicator for sustainable transport strategies in Turkey. 
The results of this study generally indicated that male respondents were more satisfied than female counterparts. The significance of gender variable shows consistency with other earlier studies (Tyrinopoulos and Antoniou, 2008; Al-Atawi and Saleh, 2014; Dölarslan, 2014; Román et al., 2014). On the other hand, the results revealed that low educated respondents were more likely to be very satisfied than higher educated respondents and this result is consistent with a very recent study (Dölarslan, 2014) conducted in another Turkish sample. Urban users were generally more likely to be very satisfied than rural users in terms of current residence variable. This results is consistent with prior studies (Kroesen 2014; Román et al., 2014). As May (2013) stated, urban areas and their populations are expected to grow substantially over the period to 2050. Therefore, keeping urban users' transport satisfaction at a respectable level may be automatically a very effective sustainable transport policy for the future. According to estimation results, respondents working in private sector were more satisfied than unemployed respondents consistent with earlier work (Al-Atawi and Saleh, 2014). Nonetheless, Filipović et al. (2009) underlined the higher satisfaction of retired passengers. Their results also seem to be similar to the outcome of some heterogeneous choice models for several transport services.

A recent study concerning the transport demand in Turkey (Haldenbilen and Ceylan, 2005) pointed out the overwhelmingly increasing demand for transport and suggested the restricted private car use or possible transfer of road transport users to alternative transport services such as rail transport. In parallel with these useful recommendations, Turkey's Sustainable Development Report (2012) has declared that decreasing the passenger road transport traffic volume under $72 \%$ until 2023 was one of the main objectives of the Turkish government in terms of sustainable transport. Another Turkish Ministry of Transport (2010) report also addressed alternative transport systems by sustaining a convenient balance among them. Other suggestions of the corresponding report were the effective use of information and communication technologies for transport services, overcoming the financial barriers of providing transport services, and improved transport infrastructure for better comfort and increased safety. These suggestions may be associated with improved smart transport systems especially in metropolitan areas.

The introduction of high-speed railway project in 2009 may be considered an important step as an implementation of sustainable transport policy and the possible transfer between several transport services in Turkey. To this end, the rail transport estimation results of this study may be valuable for future transport strategies about passenger transfer from other transport services. The policy makers may pay special attention to higher educated, rural, female and/or unemployed users' satisfaction with rail transport services and encourage them to use rail transport services. Thus, one of the most important goals on sustainable transport, namely the increasing use of alternative transport systems, may be substantially and successfully achieved. Further sustainable policy efforts for other transport services may also capture the expectations of dissatisfied users as found in this study.

The outcome of this study is limited to sample size and demographic attributes of various transport users between 2011 and 2012 due to the availability of the data. The data of further years may be continuously analysed that will enable to benchmark the estimation results. Further research may consider the perceived quality of transport services in many dimensions including comfort, accessibility, safety, privacy, etc. Particularly, the Turkish Statistical Institute may encourage respondents to give information about their income levels and may provide more specialized user satisfaction surveys involving more detailed questions about quality of transport services along with demographic characteristics. More specifically, perceived quality of services for disabled citizens may be examined in Turkey that seems to be an important future research. Some ordered response models employed in this study such as heterogeneous choice model and partial constrained generalized ordered logit models are more parsimonious models in terms of heterogeneity. Besides, future research may perform unordered response models such as multinomial and mixed models, especially for heterogeneous travel choice research when the categorical data are not ordered in nature. 


\section{References}

1. Al-Atawi, A. and Saleh, W. (2014) Travel behaviour in Saudi Arabia and the role of social factors. Transport, 29(3), 269-277. DOI: 10.3846/16484142.2014.913199

2. Beirão, G. and Sarfield Cabral, J. A. (2007). Understanding attitudes towards public transport and private car: A qualitative study. Transport Policy, 14(6), 478-489. DOI: 10.1016/j.tranpol.2007.04.009

3. Borooah, V. K. (2002) Logit and Probit: Ordered and Multinomial Models. Thousand Oaks, California: SAGE Publications.

4. Brant, R. (1990). Assessing probability in the proportional odds model for ordinal logistic regression. Biometrics, 46(4), 1171-1178. DOI: 10.2307/2532457

5. Cascetta, E. and Cartenì, A. (2014) A quality-based approach to public transportation planning: Theory and a case study. International Journal of Sustainable Transportation, 8(1), 84-106. DOI: 10.1080/15568318.2012.758532

6. Celik, E., Aydin, N. and Taskin Gumus, A. (2014) A multiattribute customer satisfaction evaluation approach for rail transit network: A real case study for Istanbul, Turkey. Transport Policy, 36, 283293. DOI: 10.1016/j.tranpol.2014.09.005

7. Celik, E., Bilisik, O. N., Erdogan, M., Taskin Gumus, A. and Baracli, H. (2013) An integrated novel interval type-2 fuzzy MCDM method to improve customer satisfaction in public transportation for Istanbul. Transportation Research Part E: Logistics and Transportation Review, 58, 28-51. DOI: 10.1016/j.tre.2013.06.006

8. Cirillo, C., Eboli, L. and Mazzulla, G. (2014) On the asymmetric user perception of transit service quality. International Journal of Sustainable Transportation, 5(4), 216-232. DOI: 10.1080/15568318.2010.494231

9. Cirit, F. (2014) Sustainable urban transport policies and comparison of mass transit systems, Planning expertise thesis, General Directorate of Economic sectors and Coordination, Turkish Ministry of Development.

10. dell'Olio, L., Ibeas, A. and Cecín, P. (2010) Modelling user perception of bus transit quality. Transport Policy, 17(6), 388-397. DOI: 10.1016/j.tranpol.2010.04.006

11. De Oña, R., Eboli, L. and Mazzulla, G. (2014) Key factors affecting rail service quality in the Northern Italy: a decision tree approach. Transport, 29(1), 75-83. DOI: 10.3846/16484142.2014.898216

12. Diana, M. (2012) Measuring the satisfaction of multimodal travelers for local transit services in different urban context. Transportation Research Part A: Policy and Practice, 46(1), 1-11. DOI: 10.1016/j.tra.2011.09.018

13. Dölarslan, E. S. (2014) Assessing the effects of satisfaction and value on customer loyalty behaviors in service environments: High-speed railway in Turkey as a case study. Management Review Research, 37(8), 706-727. DOI: .1108/MRR-06-2013-0152

14. Eboli, L. and Mazzulla, G. (2007) Service quality attributes affecting customer satisfaction for bus transit. Journal of Public Transportation, 10(3), 21-34. Retrieved from http://www.nctr.usf.edu/wpcontent/uploads/2010/03/JPT-10-3-Eboli.pdf

15. Eboli, L. and Mazzulla, G. (2011) A methodology for evaluating transit service quality based on subjective and objective measures from the passenger's point of view. Transport Policy, 18(1), 172181. DOI: 10.1016/j.tranpol.2010.07.007

16. Feng, C. M. and Hsieh, C. H. (2009) Implications of transport diversity for quality of life. Journal of Urban Planning and Development, 135(1), 13-18. DOI: 10.1061/(ASCE)0733-9488(2009)135:1(13)

17. Fu, V. (1998). Estimating generalized ordered logit models. Stata Technical Bulletin, 44, 27-30. Stata technical bulletin reprints, Vol. 8, College Station, Tex.: Stata Press, 160-164. Retrieved from http://stata-press.com/journals/stbcontents/stb44.pdf

18. Filipović, S., Tica, S., Živanović, P. and Milovanović, B. (2009) Comparative analysis of the basic features of the expected and perceived quality of mass passenger public transport service in Belgrade. Transport, 24(4), 265-273. DOI: 10.3846/1648-4142.2009.24.265-273

19. Garrido, C., de Oña, R. and de Oña, J. (2014) Neural networks for analyzing service quality in public transportation. Expert Systems with Applications, 41(15), 6830-6838. DOI: 10.1016/j.eswa.2014.04.045

20. Grujičić, D., Ivanović, I., Jović, J. and Đorić, V. (2014). Customer perception of service quality in public transport. Transport, 29(3), 285-295. DOI: 10.3846/16484142.2014.951685

21. Gulhan, G., Ceylan, H., Baskan, O. and Ceylan, H. (2014) Using potential accessibility measure for urban public transportation planning: A case study of Denizli, Turkey. Promet - Traffic \& Transportation, 26(2), 129-137. DOI: 10.7307/ptt.v26i2.1238 
22. Fiorio, C. V., Florio, M. and Perucca, G. (2013) User satisfaction and the organization of local public transport: Evidence from European cities. Transport Policy, 29, 209-218. DOI: 10.1016/j.tranpol.2013.06.004

23. Haldenbilen, S. (2003). Evaluation of sustainable transport indicators for Turkey based on genetic algorithm approach, $\mathrm{PhD}$ thesis, Institute of Science and Technology, Pamukkale University, Denizli (in Turkish).

24. Haldenbilen, S. and Ceylan, H. (2005) Transport demand management in Turkey: A genetic algorithm approach. Transportation Planning and Technology, 28(6), 403-426. DOI: 10.1080/03081060500515507

25. Jain, S., Aggarwal, P., Kumar, P., Singhal, S. and Sharma, P. (2014) Identifying public preferences using multi-criteria decision making for assessing the shift of urban commuters from private to public transport: A case study of Delhi. Transportation Research Part F: Traffic Psychology and Behaviour, 24, 60-70. DOI: 10.1016/j.trf.2014.03.007

26. Kroesen, M. (2014). Modeling the behavioral determinants of travel behavior: An application of latent transition analysis. Transport Research Part A: Policy and Practice, 65, 56-67. DOI: 10.1016/j.tra.2014.04.010

27. Lai, W. T. and Chen, C. F. (2011) Behavioral intentions of public transit passenger-The roles of service quality, perceived value, satisfaction and involvement. Transport Policy, 18(2), 318-325. DOI: $10.1016 /$ j.tranpol.2010.09.003

28. Litman, T. (2011) Developing indicators for comprehensive and sustainable transport planning. Victoria Transport Policy Institute, BC, Canada. Available from http://www.vtpi.org/sus_tran_ind.pdf

29. Litman, T. (2014) Well measured: Developing indicators for sustainable and livable transport planning. Victoria Transport Policy Institute, BC, Canada. Available from http://www.vtpi.org/wellmeas.pdf

30. Long, J. S. and Freese, J. 2001. Regression Models for Categorical Dependent Variables using Stata. College Station, Texas: Stata Press.

31. May, A. D. (2013) Urban transport and sustainability: The key challenges. International Journal of Sustainable Transportation, 7(3), 170-185. DOI: 10.1080/15568318.2013.710136

32. Mokonyama, M. and Venter, C. (2013) Incorporation of customer satisfaction in public transport contracts - A preliminary analysis. Research in Transportation Economics, 39(1), 58-66. DOI: 10.1016/j.retrec.2012.05.024

33. Pantouvakis, A. and Lymperopoulos, K. (2008) Customer satisfaction and loyalty in the eyes of new and repeat customers. Managing Service Quality: An International Journal, 18(6), 623-643. DOI: 10.1108/09604520810920103

34. Paulley, N., Balcombe, R., Mackett, R., Titheridge, H., Preston, J., Wardman, M., Shires, J. and White, P. (2006) The demand for public transport: The effects of fares, quality of service, income and car ownership. Transport Policy, 13(4), 295-306. DOI: 10.1016/j.tranpol.2005.12.004

35. Powers, D. A. and Xie, Y. (2000) Statistical Methods for Categorical Data Analysis. San Diego, California: Academic Press.

36. Quddus, M. A., Wang, C. and Ison, S. G. (2010) Road traffic congestion and crash severity: Econometric analysis using ordered response models. Journal of Transportation Engineering, 136(5), 424-435. DOI: 10.1061/(ASCE)TE.1943-5436.0000044

37. Redman, L., Friman, M., Gärling, T. and Hartig, T. (2013) Quality attributes of public transport that attract car users: A research review. Transport Policy, 25, 119-127. DOI: 10.1016/j.tranpol.2012.11.005

38. Román, C., Martín, J. C. and Espino, R. (2014) Using stated preferences to analyze the service quality of public transport. International Journal of Sustainable Transportation, 8(1), 28-46. DOI: $10.1080 / 15568318.2012 .758460$

39. Shay, E. and Khattak, A. J. (2010) Toward sustainable transport: Conventional and disruptive approaches in the US context. International Journal of Sustainable Transportation, 4(1), 14-40. DOI: $10.1080 / 15568318.2012 .758523$

40. Stathopoulos, A. and Marcucci, E. (2014) De gustibus disputandum est: Non-linearity in public transportation service quality evaluation. International Journal of Sustainable Transportation, 8(1), 47-68. DOI: 10.1080/15568318.2012.758523

41. Steg, L. and Gifford, R. (2005) Sustainable transportation and quality of life. Journal of Transport Geography, 13(1), 59-69. DOI: 10.1016/j.jtrangeo.2004.11.003 
42. St-Louis, E., Manaugh, K., van Lierop, D. and El-Geneidy, A. (2014) The happy commuter: A comparison of commuter satisfaction across modes. Transportation Research Part F: Traffic Psychology and Behaviour, 26, 160-170. DOI: 10.1016/j.trf.2014.07.004

43. Too, L. and Earl, G. (2010) Public transport service quality and sustainable development: a community stakeholder perspective. Sustainable Development, 18(1), 51-61. DOI: 10.1002/sd.412

44. Tyrinopoulos, Y. and Antoniou, C. (2008). Public transit user satisfaction: Variability and policy implications, Transport Policy, 15(4), 260-272. DOI: 10.1016/j.tranpol.2008.06.002

45. Turkish General Directorate of State Airports Authority. (2014a) 2013 Annual report. Ankara: Turkey.

46. Turkish General Directorate of State Airports Authority. (2014b) Passenger traffic (arrivaldeparture). Ankara: Turkey.

47. Turkish Ministry of Development. (2012) Turkey's sustainable development report: Claiming the future, 2012. Ankara: Turkey.

48. Turkish Ministry of Transport. (2010). Türkiye ulaşım ve iletişim stratejisi: Hedef 2023. Ankara: Turkey (in Turkish).

49. Turkish Ministry of Transport, Maritime Affairs and Communications. (2013a) Ulaşan ve erişen Türkiye, 2013. Ankara: Turkey (in Turkish).

50. Turkish Ministry of Transport, Maritime Affairs and Communications. (2013b) Istatistiklerle ulaştırma denizcilik ve haberleşme. Ankara: Turkey (in Turkish).

51. TurkStat. (2012) 2011 Life Satisfaction Survey dataset. Turkish Statistical Institute, Ankara: Turkey.

52. TurkStat. (2013) 2013 Life Satisfaction Survey dataset. Turkish Statistical Institute, Ankara: Turkey.

53. TurkStat. (2014a) Motorlu kara yaşıtları haber bülteni, A ğustos 2014. Ankara: Turkey (in Turkish)

54. TurkStat. (2014b) The number of freights and passengers in local ports. Ankara: Turkey.

55. Washington, S. P., Karlaftis, M. G. and Mannering, F. L. (2003) Statistical and Econometric Methods for Transportation Data Analysis. New York: Chapman \& Hall/CRC. .

56. Williams, R. (2006). Generalized ordered logit/partial proportional odds models for ordinal dependent variables. The Stata Journal, 6(1), 58-82. Retrieved from http://www.statajournal.com/article.html?article=st0097

57. Williams, R. (2010) Fitting heterogeneous choice models with oglm, The Stata Journal, 10(4), 540567. Retrieved from http://www.stata-journal.com/article.html?article=st0208 